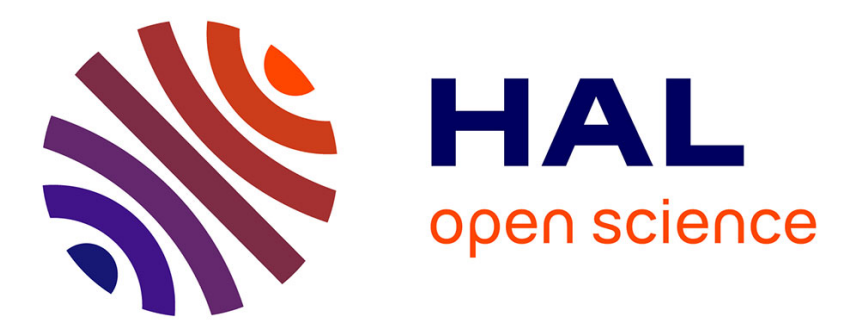

\title{
Short range order and network connectivity in amorphous AsTe3 : a first principles, machine learning, and XRD study
}

Gaëlle Delaizir, Andrea Piarristeguy, Annie Pradel, Olivier Masson, Assil Bouzid

\section{To cite this version:}

Gaëlle Delaizir, Andrea Piarristeguy, Annie Pradel, Olivier Masson, Assil Bouzid. Short range order and network connectivity in amorphous AsTe3: a first principles, machine learning, and XRD study. Physical Chemistry Chemical Physics, 2020, 22 (43), pp.24895-24906. 10.1039/d0cp03383a . hal03001599

\section{HAL Id: hal-03001599 \\ https://hal.science/hal-03001599}

Submitted on 21 Nov 2020

HAL is a multi-disciplinary open access archive for the deposit and dissemination of scientific research documents, whether they are published or not. The documents may come from teaching and research institutions in France or abroad, or from public or private research centers.
L'archive ouverte pluridisciplinaire HAL, est destinée au dépôt et à la diffusion de documents scientifiques de niveau recherche, publiés ou non, émanant des établissements d'enseignement et de recherche français ou étrangers, des laboratoires publics ou privés. 


\section{PCCP}

ARTICLE TYPE

Cite this: DOI: $00.0000 / x x x x x x x x x x$

Accepted Date

DOI: $00.0000 / x x x x x x x x x x$
Short range order and network connectivity in amorphous $\mathrm{AsTe}_{3}$ : A first principles, machine learning, and XRD study

\author{
Gaëlle Delaizir, ${ }^{a} \dagger$ Andrea Piarristeguy, ${ }^{b}$ Annie Pradel, ${ }^{b}$, Olivier Masson, ${ }^{a}$ and Assil Bouzid ${ }^{a} \ddagger$
}

The atomic scale structure of amorphous $\mathrm{AsTe}_{3}$ is investigated through $\mathrm{X}$-ray diffraction, firstprinciples molecular dynamics (FPMD), and machine learning interatomic potentials (ML-GAP) obtained by exploiting the ab-initio data. We obtain a good agreement between the measured and modelled diffraction patterns. Our FPMD results show that $\mathrm{As}$ and $\mathrm{Te}$ obey the 8-N rule with an average coordination numbers of 3 and 2, respectively. We find that small fractions of under and over coordinated As and Te atoms occur in the amorphous phase with about 6\% (FPMD), and 13\% (ML-GAP) of 3-fold Te. As is found at the center of pyramidal structures predominantly linked through $\mathrm{Te}_{n}$ chains rather than rings. Despite the low As concentration in $\mathrm{AsTe}_{3}$, its local environment feature a very high chemical disorder that manifests through the occurrence of homopolar bonds including at least $57 \%$ of As atoms.

\section{Introduction}

Chalcogenide glasses are attractive materials for a wide range of technologically relevant applications including chemical sensors, medical and environmental devices, optical lenses, optical fibers, optoelectronic devices, Li-ion solid-state batteries and phase change memories.10 Most of chalcogenide glasses exhibit a semi-conducting behavior owing to their peculiar electronic band-structure where spatially localized electronic states contribute to the electrical transport. While this feature is a main drawback for practical applications in semiconductor and optoelectronic areas, it is of interest for applications in the thermoelectric domain where a high thermopower and a very low thermal conductivity are highly required.

Among thermoelectric chalcogenide materials, Te-based compounds show outstanding performances. Within this family, crystalline $\mathrm{Bi}_{2} \mathrm{Te}_{3}$ is the best thermoelectric material operating at room temperature. ${ }^{11 \mid 12}$ In addition, its isostructural $\beta-\mathrm{As}_{2} \mathrm{Te}_{3}$ compound shows remarkable thermoelectric properties. $\frac{13}{13}$ The search for high performing thermoelectric materials led recently to the development of a new crystalline compound with composition $\mathrm{AsTe}_{3}$, that exhibits a very low thermal conductivity and an interesting figure of merit (ZT) peak value of 0.3 at $405 \mathrm{~K}$, indicative of its thermoelectric performance. 14 An interesting feature of this compound is that it was exclusively synthesized from

\footnotetext{
${ }^{a}$ Institut de Recherche sur les Céramiques (IRCER), UMR CNRS 7315-Université de Limoges, France.

${ }^{b}$ Address, ICGM, Univ Montpellier, CNRS, ENSCM, Montpellier, France.

$\ddagger$ assil.bouzid@unilim.fr

$\dagger$ gaelle.delaizir@unilim.fr
}

the full and congruent crystallization of the parent glass. ${ }^{14}$ Thus, while only the crystalline phase is of interest for thermoelectric applications, a better control of the synthesis and stability of the cristalline phase require a fundamental understanding of the parent glassy $\mathrm{AsTe}_{3}$. In this work, we focus on the structural properties of the amorphous phase of $\mathrm{AsTe}_{3}$.

Within the As-Te family, several glassy $\mathrm{As}_{x} \mathrm{Te}_{1-x}(0.2 \leq \mathrm{x} \leq$ 0.8 ) compositions were synthesized and their properties investigated. $15-27$ However, all the studied compositions were found to be thermally unstable and require either fast quenching rates ${ }^{14}$ or conventional quenching of very small quantities to retain their vitreous state. 15 The atomic scale picture of As-Te systems remains elusive and various descriptions of the network connectivity and the short range order are provided in the literature. $15+20 \mid 28$ In particular, Mössbauer spectroscopy revealed the presence of three fold Te environments in Te-rich compounds. 28 This result was extended by Raman measurements which proposed that a large fraction of three fold Te occurs in the entire compositional range of As atoms. $\frac{15}{15}$ On the opposite side, recent X-ray diffraction experiments combined with X-ray absorption fine structure (EXAFS) spectroscopy and reverse Monte Carlo simulation, 17 in addition to high-resolution two-dimensional projection magic-angleturning ${ }^{125} \mathrm{Te}$ nuclear magnetic resonance (NMR) spectroscopy revealed that coordination number of As and Te obey the 8-N rule over the entire compositional range. 16 Moreover, most of these studies reported the presence of a significant chemical disorder irrespective of the Te concentration in the material.

In the present work, we resort to first-principles molecular dynamics (FPMD) combined with, machine learning (ML) and Xray diffraction $(\mathrm{XRD})$ to investigate the atomic scale structural 
organization of glassy Te-rich $\mathrm{AsTe}_{3}$ compound. FPMD techniques have shown a high record of reliability in investigating a wide class of chalcogenides including S, Se and Te-based materials. 29.35 We focus our efforts solely on the amorphous $\mathrm{AsTe}_{3}$ compound, as a good understanding of its structure provides direct insights into that of the metastable undoped crystalline $\mathrm{AsTe}_{3}$ thermoelectric material.

The paper is organized as follows. Synthesis and sample characterisation are given in Section 2 . In Sec. 3, we provide a description of the FPMD methodology (Sec. 3.1) and machine learning approach (Sec. 3.2) employed in this work. Results, including real and reciprocal space properties, atomic local environment and network connectivity, are presented in Sec. 4. The conclusions of our work are drawn in Sec.5.

\section{Sample preparation and caracterisation}

\section{1 $\mathrm{AsTe}_{3}$ Synthesis}

$\mathrm{AsTe}_{3}$ was synthesized from high-purity elemental As (Goodfellow, 99.99\%) and Te $(5 \mathrm{~N}+, 99.99 \%)$. Approximately $3 \mathrm{~g}$ of stoichiometric powders were placed in a cylindrical silica ampule (6 $\mathrm{mm}$ inner diameter and $1 \mathrm{~mm}$ thick) sealed under secondary vacuum $\left(10^{-5}\right.$ mbar). The tube was subsequently heated in a vertical furnace up to $1123 \mathrm{~K}$, with a low heating rate of $9 \mathrm{Kh}^{-1}$, held at this temperature for $2 \mathrm{~h}$, and finally quenched in a salt-ice-water mixture. The obtained ingot was crushed into small pieces, remelted then quenched using the twin roller quenching technique. To this end, the resulting pieces were placed in a quartz tube with a drilled hole at the bottom of the tube. These pieces were heated using a radio frequency induction furnace, which allowed their temperature to rise very rapidly. An over pressure using an Ar gas jet was introduced forcing small droplets of the melt to flow through the hole in between the rotating twin rollers. Amorphous quenched flakes (typically $\sim 40 \mu \mathrm{m}$ in thickness and $\sim 1 \mathrm{~cm}^{2}$ in surface area) were released by the rollers and dropped into an $\mathrm{Al}$ collector. The entire process was carried out inside a glove box kept under Ar atmosphere (for more details on the experimental procedure see Pradel et al. [ 36]).

\subsection{Physico-chemical caracterisations}

The chemical composition of the amorphous flakes after twin roller quenching was checked by SEM-EDS using a ZEISS EVO HD15 equipment. Different flakes and zones were analyzed in order to obtain information on their chemical homogeneity. We find mean atomic percentages of $24.3 \pm 1.0$ and $75.7 \pm 1.0$ for As and Te in the amorphous flakes, respectively. This result is in agreement with the target stoichiometric composition $\mathrm{AsTe}_{3}$. Glass transition and crystallization temperatures were recorded using Differential Scanning Calorimetry (DSC) AQ20 (TA Instruments) with a heating rate of $10 \mathrm{~K} / \mathrm{min}$ as displayed in Figure 1 . The DSC thermogram shows that $\mathrm{AsTe}_{3}$ glass exhibits a glass transition temperature $\left(\mathrm{T}_{g}\right)$ and a temperature of the onset of the crystallization $\left(\mathrm{T}_{c}\right)$ at $367 \mathrm{~K}$ and $413 \mathrm{~K}$, respectively. These temperatures are quite similar to the values obtained by J. Cornet et al. 19 on similar As-Te glasses. We note that the difference between $\mathrm{T}_{c}$ and $\mathrm{T}_{g}$ is about $46 \mathrm{~K}$, which indicates a rather limited thermal stability of this glass 37

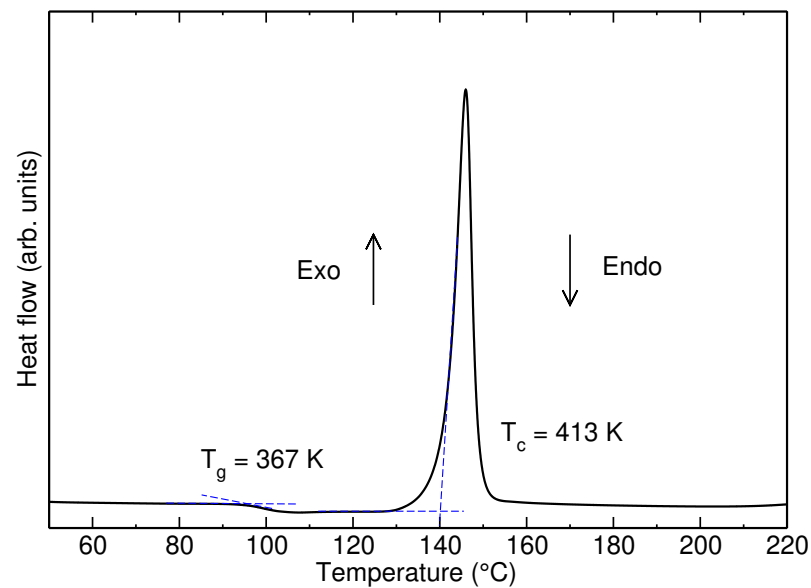

Fig. 1 Differential scanning calorimetry thermogram of amorphous $\mathrm{AsTe}_{3}$. The glass transition temperature $\mathrm{T}_{g}$ and the crystallization temperature $\mathrm{T}_{c}$ are provided.

Furthermore, the density $\left(\rho_{0}\right)$ of $\mathrm{AsTe}_{3}$ powder was determined using an helium pycnometer (Micromeritics) and was found to be $5.639 \mathrm{gcm}^{-3}$, close to the value reported by J. Cornet et al. 19

Insights into the structure of amorphous $\mathrm{AsTe}_{3}$ sample was obtained throught X-ray total scattering. Measurements of the total X-ray structure factor $S_{T}(k)$ were performed with a dedicated laboratory setup based on a Bruker D8 advance diffractometer ( $\lambda=0.559422 \AA$ ) equipped with a silver sealed tube and rapid LynxEye XE-T detector. This setup was modified in order to maximize collected intensities, to minimize spurious signal from empty environment and to get good counting statistics up to a large scattering vector length of $21.8 \AA^{-1}$. Raw $S_{T}(k)$ data were corrected, normalized and Fourier transformed using a homemade software $\frac{38}{38}$ in order to get the atomic Pair Distribution Function (PDF) of the sample $\left(g_{\mathrm{T}}(r)\right)$. The corrections included capillary, empty environment and Compton scatterings, fluorescence, absorption and polarization. The obtained $S_{T}(k)$ and $g_{\mathrm{T}}(r)$ are plotted in Figures 3 and 4 respectively and will be discussed in section 4.1 .

\section{Computational details}

\subsection{First-principles molecular dynamics simulations}

In this work, the electronic structure is described in the framework of density functional theory. We resort to the Becke Lee, Yang and Parr (BLYP) 39.40 functional to describe the exchange and correlation parts of the total energy. The choice of BLYP is motivated by its high record of reliability when applied for chalcogenide materials. $29-35$ In particular, BLYP demonstrated a superior performances compared to Perdew, Burke, and Ernzerhof 41 (PBE) functional when modelling Te-based, $\mathrm{GeTe}_{4}$, binary chalcogenide system, $\underline{42}$ and led to very good structural models for ternary systems including $\mathrm{Ge}_{2} \mathrm{Sb}_{2} \mathrm{Te}_{5}, 43 \mathrm{Ga}_{4} \mathrm{Sb}_{6} \mathrm{Te}_{3} 44$ and, $\mathrm{Ga}_{10} \mathrm{Ge}_{15} \mathrm{Te}_{75} \cdot{ }^{45}$ The core-valence interactions are described by normconserving Troullier and Martins pseudopotentials. 46 The wave functions of the valence electrons were expanded in a plane- 
wave basis set defined by a kinetic energy cutoff of 40 Ry. The Brillouin zone was sampled at the $\Gamma$ point. In the case of Tebased chalcogenides, it has been shown that van der Waals (vdW) corrections play an essential role in reproducing structural models featuring a good agreement with experiments. ${ }^{42 / 45}$ Therefore, we account for long-range dispersion forces through the DFT-D2 correction term due to Grimme. ${ }^{47}$ We resort to the Car-Parrinello molecular dynamics as implemented in the CPMD code. $\frac{48}{} \mathrm{~A}$ fictitious electron mass of 500 a.u. and a time step of $\Delta t=0.12 \mathrm{fs}$ are used to integrate the equations of motion, ensuring a good control of the conserved quantity. A Nosé-Hoover thermostat chain 49 is implemented to ensure the temperature control of the ionic degrees of freedom and a similar thermostat due to Blöchl and Parrinello 53 ensures the control of the temperature of the fictitious electronic degrees of freedom.

We model $\mathrm{AsTe}_{3}$ using a periodic cubic cell containing 240 atoms (60 As and $180 \mathrm{Te}$ ) at the experimental density of 5.639 $\mathrm{gcm}^{-3}$ (cell side length $20.07 \AA$ ). The initial configuration was built by selecting 240 atomic positions from an old binary chalcogenide $\left(\mathrm{GeSe}_{4}{ }^{54}\right)$ and performing an atom substitution. The system was then subject to a thermal annealing cycle as follows: 10 $\mathrm{ps}$ at $\mathrm{T}=300 \mathrm{~K}, 10 \mathrm{ps}$ at $\mathrm{T}=500 \mathrm{~K}, 50 \mathrm{ps}$ at $\mathrm{T}=650 \mathrm{~K}, 50 \mathrm{ps}$ at $\mathrm{T}=$ $500 \mathrm{~K}$ and $25 \mathrm{ps}$ at $300 \mathrm{~K}$. We note that both atomic species reached high mobility during the run at the melting temperature $(\mathrm{T}=650$ $\mathrm{K})$ characterised by a liquid-like diffusion coefficients $\left(\mathrm{D}_{\mathrm{As}}=3.5\right.$ $10^{-5} \mathrm{~cm}^{2} / \mathrm{s}$ and $\mathrm{D}_{\mathrm{Te}}=4.210^{-5} \mathrm{~cm}^{2} / \mathrm{s}$ ). As such As and Te have undergone displacements several times larger than interatomic distances which ensures that no memory remains from the used initial configuration. The residual stress is calculated for the final structure obtained at $\mathrm{T}=300 \mathrm{~K}$ and was found to be equal to $\sim$ $0.8 \mathrm{GPa}$. Such a residual stress has been shown to alter the equilibrium structural properties of chalcogenide systems ${ }^{55}$ and was eliminated by relaxing the cell following the procedure described in Ref. 55 yielding a slightly lower glass density of $5.45 \mathrm{gcm}^{-3}$ (cell side length $20.3 \AA$ ). The obtained stress-free structure was then further annealed at $\mathrm{T}=300 \mathrm{~K}$ during $43 \mathrm{ps}$ at an internal stress lower than $0.05 \mathrm{GPa}$. The final $20 \mathrm{ps}$ of this trajectory were used to compute statistical averages of the structural properties of glassy $\mathrm{AsTe}_{3}$ presented in this work.

In order to achieve an accurate and unbiased description of the network connectivity, we rely on the formalism of the maximally localized Wannier functions (MLWF) .5657 Within this method, a Wannier function $\left(w_{n}(\mathbf{r})\right)$ corresponds to the localization of two electrons and its center (W) gives its average position. As such, MLWF leads to an accurate and compact real-space representation of the electronic structure, which we can use to define chemical bonds and lone pairs. In practice, $w_{n}(\mathbf{r})$ and their corresponding centers $\mathrm{W}$ are obtained by an on the fly unitary transformation of the Kohn-Sham orbitals $\psi_{i}(\mathbf{r})$ under the constraint of minimizing the spatial extension (spread, $\Omega$ ) of the resulting $w_{n}(\mathbf{r})$ as follows:

$$
\Omega=\sum_{n}\left(\left\langle w_{n}\left|\mathbf{r}^{2}\right| w_{n}\right\rangle-\left\langle w_{n}|\mathbf{r}| w_{n}\right\rangle^{2}\right)
$$

Wannier functions were computed on top of 30 to 100 configurations selected along the last $20 \mathrm{ps}$ at $\mathrm{T}=300 \mathrm{~K}$ and their centers were used to investigate the network connectivity (occurrence of
Table 1 GAP model and hyper-parameters of the SOAP and 2_body kernels.

\begin{tabular}{ccc}
\hline \hline Atomic environment kernel & SOAP & 2 _body \\
$r_{\text {cut }}$ & 6 & 6 \\
$r_{\Delta}$ & 1 & 1 \\
$\sigma_{\omega}$ & 1.0 & 0.0995 \\
$\theta$ & - & 1 \\
$\sigma_{\text {atom }}$ & 0.5 & - \\
$\xi$ & 4 & - \\
$n_{\text {max }}$ & 8 & - \\
$l_{\text {max }}$ & 8 & - \\
Represenative environments & 1600 & 50 \\
\hline$\sigma_{v}^{\text {energy }}$ & $1.0 \times 10^{-3} \mathrm{eV} /$ atom \\
$\sigma_{v}^{\text {force }}$ & $5.0 \times 10^{-2} \mathrm{eV} / \AA$ \\
$\sigma_{v}^{\text {virial }}$ & $5.0 \times 10^{-2} \mathrm{eV} /$ atom \\
sparse jitter & \multicolumn{2}{c}{$10^{-12}$} \\
GAP software version & 1563528539 \\
\hline \hline
\end{tabular}

chemical bonding between neighboring atoms).

\subsection{Machine learning modelling}

Despite the predictive power of FPMD methodology, its extension to large time- and size scales is inherently associated to the explicit treatment of the electrons and of their interactions. This limitation can be overcome by resorting to machine learning (ML) to generate interatomic potentials that allows a costeffective simulations of large systems. In particular, we resort to the Gaussian Approximation Potential (ML-GAP) approach that allows to learn and reproduce smooth highly dimensional potential energy surface by interpolating DFT data. 58 In this technique, each point of the potential energy surface is written as a sum of a universal local atomic energy function $\varepsilon_{i}$ that depends on the geometry of neighbors atoms structure around atom $i$ and within a sphere of radius $r_{\text {cut }}$. Then, Gaussian regression process $\frac{6162}{16}$ is used to define a model for $\varepsilon_{i}$ as a linear combination of nonlinear Kernel functions. An extensive review of this technique is provided in Ref.60. This technique has been successfully applied to model glasses, liquids, and crystals. 63 In this work, we apply ML-GAP technique as implemented in QUIP (http://www.libatoms.org). We combine two descriptors for the representation of the atomic structure where the Smooth Overlap of Atomic Positions (SOAP) 67 is complemented by a nonparametric two-body distance descriptor in order to prevent nonphysical clustering of atoms. We train the ML-GAP potential on a data base build by extracting representative configurations from the FPMD trajectories as follows: 216, 240, and 243 configurations at $\mathrm{T}=650 \mathrm{~K}, 500 \mathrm{~K}$, and $300 \mathrm{~K}$, respectively, and 240 stressfree configurations at $\mathrm{T}=300 \mathrm{~K}$. This amounts to 225360 local environment to be fitted. In order to achieve a good accuracy of the data base, we recomputed DFT energies, forces, and stresses for all the configurations at an energy cutoff of 140 Ry. Table 1 shows the details of the GAP model and the hyper-parameters of the used descriptors.

The obtained ML-GAP potential is then used to compute ener- 

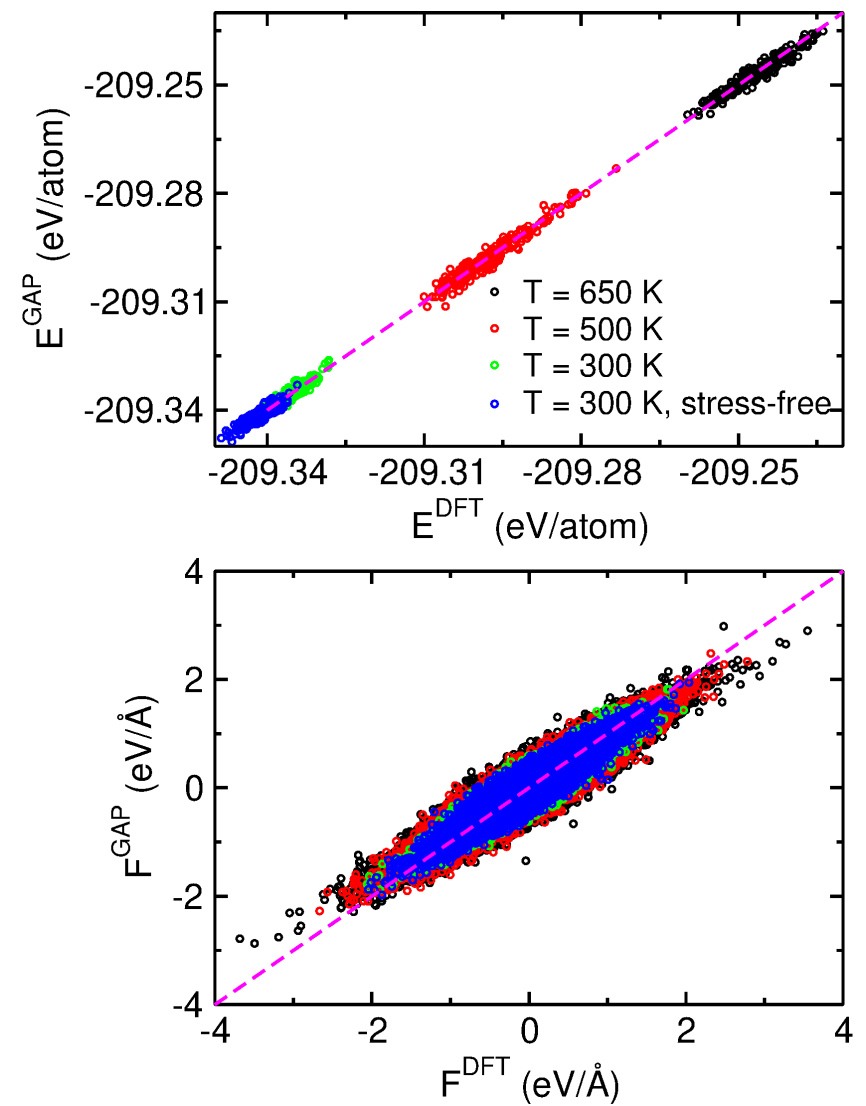

Fig. 2 Comparison between total energies (top panel) and force components (bottom panel) as obtained from ML-GAP potential and DFT calculations on the training data base.

gies and forces on the training configurations. The results presented in Figure 2 show a very good match between all the quantities within a root mean square errors of $1.25 \mathrm{meV} /$ atom for energy and $0.16 \mathrm{eV} / \AA$ for the force. Similar errors were also obtained on a validation data base made of configurations unknown to the trained potential, thereby validating the accuracy of the obtained ML-GAP interatomic potential.

Finally, we exploit the ML-GAP potential to produce a large amorphous model of $\mathrm{AsTe}_{3}$ containing 1920 atoms (480 As and $1440 \mathrm{Te}$ ). MD is carried out using the ML-GAP potential in the NVT ensemble (cell side length $40.6 \AA$ ) and with an integration time step of $0.5 \mathrm{fs}$ as implemented in the LAMMPS code $\frac{68}{6}$. As in the case of the FPMD model, the glass is obtained after a thermal annealing cycle featuring: $5 \mathrm{ps}$ at $\mathrm{T}=300 \mathrm{~K}, 5 \mathrm{ps}$ at $\mathrm{T}=500$ $\mathrm{K}, 70 \mathrm{ps}$ at $\mathrm{T}=650 \mathrm{~K}, 70 \mathrm{ps}$ at $\mathrm{T}=500 \mathrm{~K}$, and $70 \mathrm{ps}$ at $\mathrm{T}=$ $300 \mathrm{~K}$. The obtained glassy model will allow us to consolidate the FPMD structural model of $\mathrm{AsTe}_{3}$ by relaying on a large size system obtained at the DFT accuracy. When useful, results of the ML-GAP $\mathrm{AsTe}_{3}$ model are presented and discussed throughout the paper.

\section{Results}

\subsection{Total structure factor and total pair correlation function: Experiments vs Modelling}

In X-ray diffraction experiments the total X-ray structure factor $S_{T}(k)$ is defined by:

$$
S_{\mathrm{T}}(k)-1=\sum_{\alpha=1}^{2} \sum_{\beta=1}^{2} \frac{c_{\alpha} c_{\beta} f_{\alpha}(k) f_{\beta}(k)}{\left|\langle f(k)\rangle^{2}\right|}\left[S_{\alpha \beta}^{\mathrm{FZ}}(k)-1\right],
$$

where $\alpha$ and $\beta$ denote one of the two chemical species As and/or Te. $c_{\alpha}$ and $f_{\alpha}(k)$ are the atomic concentrations of species $\alpha$ (As, or Te) and its corresponding scattering factor. $S_{\alpha \beta}^{\mathrm{FZ}}(k)$ is the FaberZiman (FZ) partial structure factor and $k$ is the magnitude of the scattering vector. ${ }^{69} S_{\alpha \beta}^{\mathrm{FZ}}(k)$ partial structure factors can be directly calculated on the equilibrium trajectory by solving the Debye equation in the reciprocal space. Consequently, one can have a direct access to the $S_{\mathrm{T}}(k)$. Alternatively, $S_{\alpha \beta}^{\mathrm{FZ}}(k)$ can be obtained by Fourier transform (FFT) of the real space pair correlation functions $g_{\alpha \beta}(r)$ as follows:

$$
S_{\alpha \beta}^{\mathrm{FZ}}(k)-1=\frac{4 \pi \rho_{0}}{k} \int_{0}^{\infty} r\left[g_{\alpha \beta}(r)-1\right] \sin (k r) d r .
$$

Similarly to $S_{\mathrm{T}}(k)$ the total pair distribution function $g_{\mathrm{T}}(r)$ is often calculated using $g_{\alpha \beta}(r)$ :

$$
g_{\mathrm{T}}(r)-1 \approx \sum_{\alpha=1}^{2} \sum_{\beta=1}^{2} \frac{c_{\alpha} c_{\beta} f_{\alpha}\left(k_{0}\right) f_{\beta}\left(k_{0}\right)}{\left|\left\langle f\left(k_{0}\right)\right\rangle^{2}\right|}\left[g_{\alpha \beta}(r)-1\right] .
$$

Where the scattering factors are evaluated at some arbitrary value $k_{0}$, generally zero. While this approximation often leads to a fair representation of $g_{\mathrm{T}}(r)$, the magnitude of the first peaks can be altered, in particular for samples combining both heavy and light elements. We here resort to the method developed by Masson and Thomas ${ }^{70}$ where an exact and explicit $g_{\mathrm{T}}(r)$ expression was derived. In their formalism, $g_{\mathrm{T}}(r)$ can be obtained through a weighted linear combination of modified partial pair correlation functions where the weights are defined as the mean values of the Faber-Ziman factors over the considered reciprocal space range. In practice, the modified partial pair correlation functions are obtained by superposing on the original partial PDFs their weighted and symmetrically shifted ghost PDF functions. A full description of this method is provided in Ref. [ 70].

The measured X-ray structure factor of glassy $\mathrm{AsTe}_{3}$ is provided in figure 3. We remark that $S_{\mathrm{T}}(k)$ features a narrow first peak at about $1.8 \AA^{-1}$, followed by a much broader peaks $(\sim 3.35$ and $5.25 \AA^{-1}$ ) and rapidly damped oscillations corresponding to a structural correlation length of about $12 \AA^{-1}$. Figure 3 shows also the $\mathrm{AsTe}_{3}$ total structure factor directly calculated in the reciprocal space and that obtained by Fourier transform of the pair correlation functions of the FPMD model in addition to the FFT $S_{\mathrm{T}}(k)$ of the ML-GAP model. Furthermore, we provide the measured X-ray structure factor of glassy $\mathrm{As}_{20} \mathrm{Te}_{80}$ from Ref. [ [17]. Our results show a good agreement between FPMD/ML-GAP models and the measured data over the entire range of reciprocal space within typical statistical fluctuations. In particular, the positions and intensities of the first three peaks $\left(k<6 \AA^{-1}\right)$ are very well 


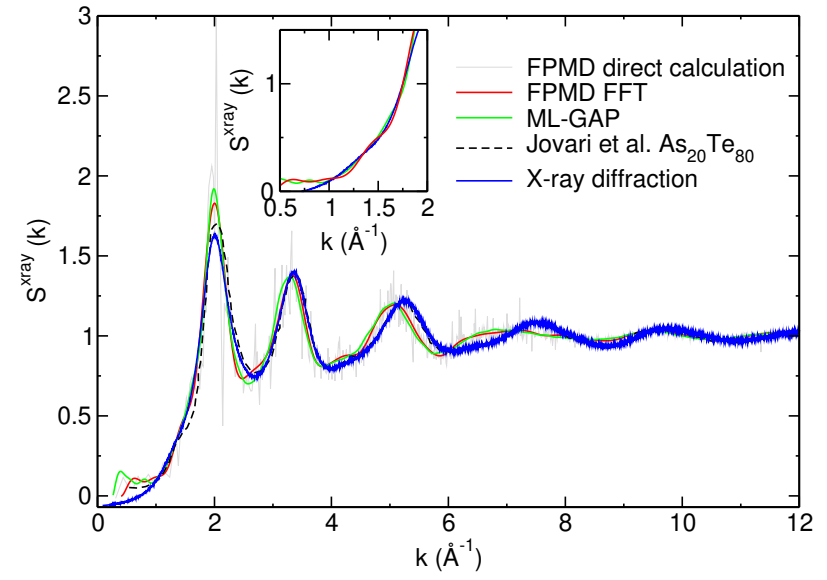

Fig. 3 Total X-ray structure factor for amorphous $\mathrm{AsTe}_{3}$ at $\mathrm{T}=300$ $\mathrm{K}$. The experimental results (blue lines) are compared to the calculated FPMD $S_{\mathrm{T}}(k)$ in the reciprocal space (gray lines) or obtained through Fourier transform of the pair correlation functions with a cutoff value $k_{\max }=21.8 \AA$ (red lines), and to the ML-GAP $S_{\mathrm{T}}(k)$ (green lines). These results are also compared to the measured $S_{\mathrm{T}}(k)$ for amorphous $\mathrm{As}_{20} \mathrm{Te}_{80}$ from Ref. [ 17] (black lines). The inset represents a zoom on the FSDP region.

reproduced. Small discrepancies occur for large $k$ values. Despite the slightly different composition, we find that $\mathrm{AsTe}_{3}$ data are in a very good agreement with those of $\mathrm{As}_{20} \mathrm{Te}_{80} .17$

Amorphous chalcogenide systems feature an intermediate range order (IRO) that manifests through the appearance of the first sharp diffraction peak (FSDP) at around $k \sim 1 \AA^{-1}$ in the total structure factor. ${ }^{7172}$ In the particular case of Ge-based chalcogenides, the IRO was correlated to the occurrence of a particular connectivity between Ge-centered tetrahedra through edge- and corner-sharing connections. ${ }^{29}$ In the case of $\mathrm{As}_{2} \mathrm{Te}_{3}$, diffraction data showed the presence of a FSDP in the amorphous phase. ${ }^{73}$ However, it appears from our results that while a small shoulder for $k$ ranging from 1 to $1.4 \AA^{-1}$ is present in the case of the FPMD model of $\mathrm{AsTe}_{3}$ (see panel in Figure 3), the ML-GAP model does not show any distinguishable feature around $1 \AA^{-1}$. The absence of a relevant FSDP reveals that the IRO is very limited in amorphous $\mathrm{AsTe}_{3}$. We note that the small peak at $k<1 \AA^{-1}$ cannot be ascribed to any structural feature as it is a Fourier transform artifact due to the finite system size. Overall, we remark that the $S_{\mathrm{T}}(k)$ obtained through FFT allows to capture all the features of the structure factor calculated directly in the reciprocal space.

Figure 4 shows the experimental $g_{\mathrm{T}}(r)$ obtained by Fourier transforming the measured $S_{\mathrm{T}}(k)$ and compared to that obtained from FPMD and ML-GAP models. The measured $g_{\mathrm{T}}(r)$ shows an overall pattern typical of amorphous materials. We distinguish a first sharp peak at $2.71 \AA$, followed by a second broader peak at 4.02 A reflecting the first and second coordination shells, respectively. Few other peaks occur at larger $r$ values, however, their intensities are rapidly damped due to the abscence of long range correlations in the amorphous phase.

Compared to experiments, the $g_{\mathrm{T}}(r)$ as obtained from the FPMD and ML-GAP models shows an excellent agreement over the entire real space range. For $r<5.4 \AA$, the measured first peak

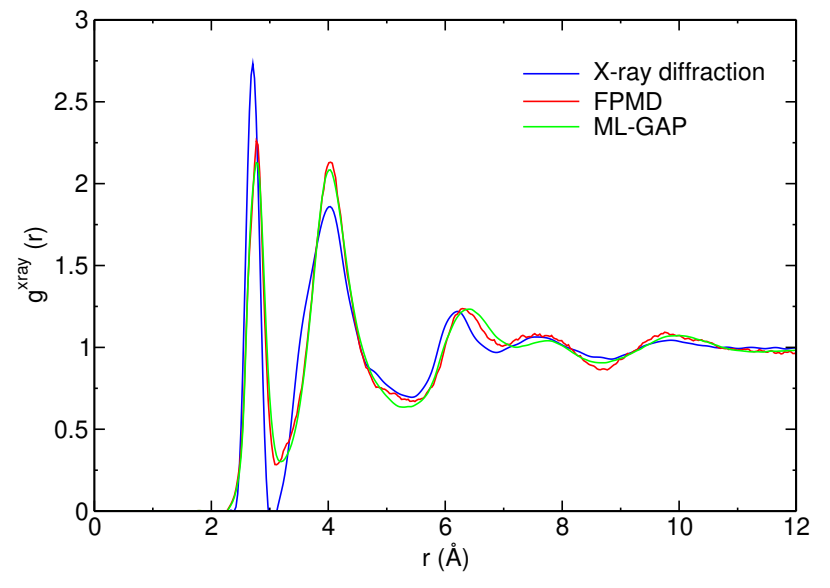

Fig. 4 Total pair correlation function for amorphous $\mathrm{AsTe}_{3}$ at $\mathrm{T}=300$ $\mathrm{K}$. The experimental results (blue lines) are compared to the FPMD calculated $S_{\mathrm{T}}(k)$ (red lines) and to ML-GAP $S_{\mathrm{T}}(k)$ (green lines).

shows a slightly higher intensity compared to the modelled one, while the opposite case occurs when looking at the second peak. We also remark that the experimental $g_{\mathrm{T}}(r)$ show a small shoulder at around $3.5 \AA$ that is not reproduced in the modelled ones. These small discrepancies are typical of statistical errors and are expected to have a very limited influence on the short range order of $\mathrm{AsTe}_{3}$. This rational is substantiated by the fact that the integral of the first peak up to the first minimum, which directly correlates to the average atomic coordination number, gives the same result for FPMD and XRD pattern. The same rational applies also to the second peak. For $r>5.4 \AA$, the FPMD and ML-GAP models reproduce the positions and intensities of the peaks occurring at medium range distances. Overall, these results provide evidences that our models allow for a very good description of the topology of glassy $\mathrm{AsTe}_{3}$ in comparison to experiments.

\subsection{Faber-Ziman partial structure factors}

In order to disentangle the various contributions of each chemical species into the network topology we consider the partial FZ structure factors as displayed in Fig. 5

Focusing on $S_{\mathrm{TeTe}}^{\mathrm{FZ}}(k)$, one can immediately remark that it follows closely the pattern of the total structure factor $S_{\mathrm{T}}(k)$ (see Fig. 3). This result is not surprising and is a direct consequence of the large concentration of Te atoms in the system, as well as their large atomic scattering factor, that leads to a dominant contribution of $S_{\mathrm{TeTe}}^{\mathrm{FZ}}(k)$ into the total structure factor as evidenced by Eq. 2 . We remark that the small discrepancies between the measured and calculated $S_{\mathrm{T}}(k)$ at $k$ larger than $6 \AA^{-1}$ observed in Figure 3 are mainly due to the Te-Te correlations. In this respect, a more accurate description of these correlation would require a higher level of theory for the description of vdW interactions within the DFT setup. Nevertheless, such discrepancies occurring at high $k$ values are expected to have a very limited impact on the local structure around Te. In the case of $S_{\mathrm{AsTe}}^{\mathrm{FZ}}(k)$ and $S_{\mathrm{AsAs}}^{\mathrm{FZ}}(k)$, we remark the occurrence of an FSDP-like peaks in between $1 \AA^{-1}$ and $1.5 \AA^{-1}$, which might be correlated with the occurrence of an intermediate range order around As atoms. Nevertheless, due to 


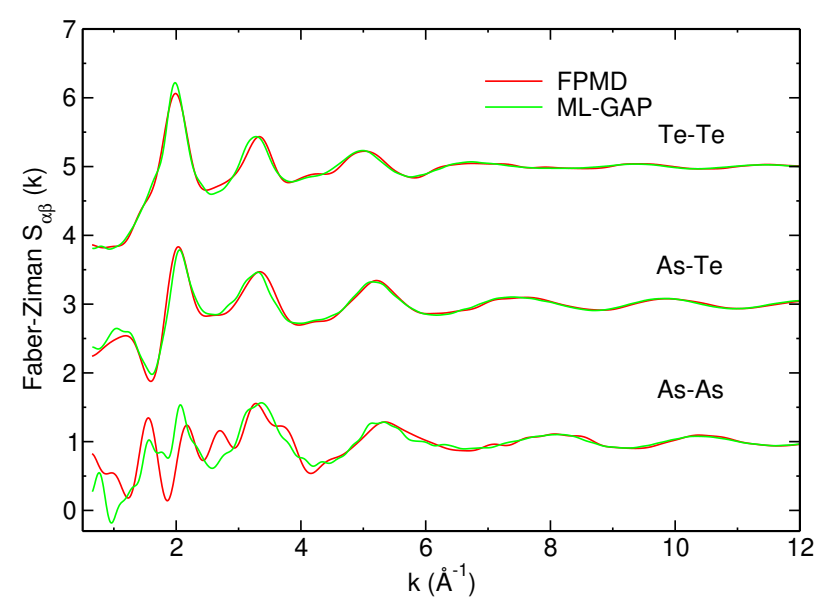

Fig. 5 The Faber-Ziman partial structure factors $S_{\mathrm{TeTe}}^{\mathrm{FZ}}(k), S_{\mathrm{AsTe}}^{\mathrm{FZ}}(k)$, and $S_{\mathrm{AsAs}}^{\mathrm{FZ}}(k)$ for amorphous $\mathrm{AsTe}_{3}$ at $\mathrm{T}=300 \mathrm{~K}$ obtained from FPMD (red lines) and ML-GAP (green lines). The curves are shifted vertically for clarity.

the small concentration of As atoms, these peaks are barely visible in the $S_{\mathrm{T}}(k)$. In addition, while the ML-GAP model shows a reduction of the fluctuations of $S_{\mathrm{AsAs}}^{\mathrm{FZ}}(k)$ for $k<4 \AA^{-1}$ it does not promote any clear peak around $1 \AA^{-1}$. Taken together, FZ partial structure factors provide evidences of the dominance of Te-Te correlations as well as a very limited IRO in amorphous $\mathrm{AsTe}_{3}$.

\subsection{Partial pair correlation functions}

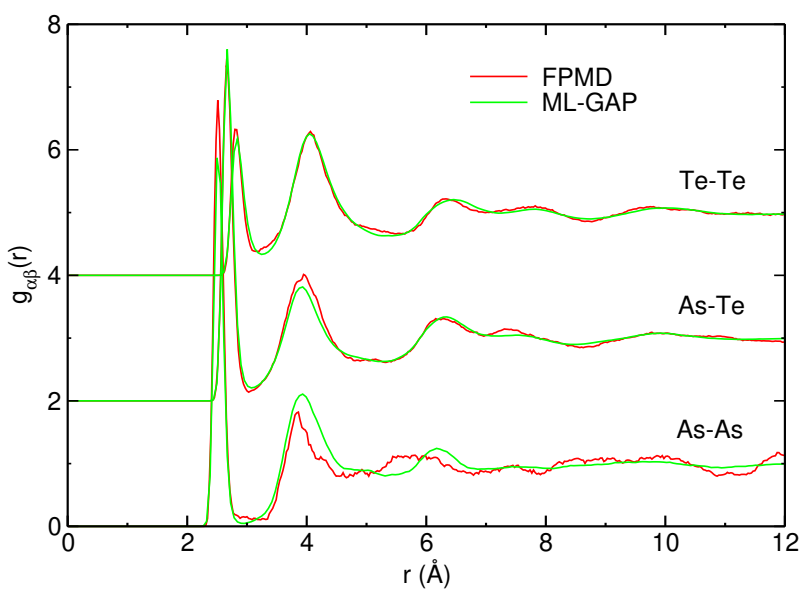

Fig. 6 The partial pair correlation functions $g_{\mathrm{TeTe}}(r), g_{\mathrm{AsTe}}(r)$, and $g_{\mathrm{AsAs}}(r)$ for amorphous $\mathrm{AsTe}_{3}$ at $\mathrm{T}=300 \mathrm{~K}$ obtained from FPMD (red lines) and ML-GAP (green lines). The curves are shifted vertically for clarity.

The atomic-scale picture of $\mathrm{AsTe}_{3}$ as described in the reciprocal space can be substantiated by investigating the real space properties of this glass. Figure 6 shows the FPMD and ML-GAP calculated partial pair correlation functions of glassy $\mathrm{AsTe}_{3}$.

$g_{\text {AsAs }}(r)$ feature a narrow first peak around $2.51 \AA$ indicative of the presence of As-As homopolar bonds in glassy $\mathrm{AsTe}_{3}$. The high intensity of this peak reflects that As homopolar bonds occurs in a relatively large fraction. For larger $r$ values, we remark that the FPMD model suffers from some statistical fluctuations due to the relatively small number of As atoms in the cell. This leads to a wide first minimum gap between the first and the second peaks in addition to the absence of a clear trend for $r>5 \AA$. Interestingly, the ML-GAP model provides a better description of the As-As correlations in real space where peaks representative of second (around $4 \AA$ ) and third (around $6.2 \AA$ ) coordination shells are well distinguished in comparison to the FPMD model. We note also a small reduction of the intensity of the first peak in the ML-GAP model.

Focusing on $g_{\mathrm{TeTe}}(r)$, we find a first peak centered around 2.81 $\AA$ indicative of homopolar Te-Te bonds and a second peak around 4.06 $\AA$ originating from the Te belonging to the second coordination shell. These two peaks feature a similar intensities due to the high concentration of Te atoms and their ability to form -Te-Te-Telinkages. In addition, one can distinguish three peaks at medium range distances ( $r>5.5 \AA$ ) that might be indicative of the presence of long $\mathrm{Te}_{n}$ chains with $n>3$. Finally, $g_{\mathrm{AsTe}}(r)$ shows a first sharp peak around $2.65 \AA$. For $r$ larger than $3 \AA$ we remark some similarities between $g_{\mathrm{AsTe}}(r)$ and $g_{\mathrm{TeTe}}(r)$, which can be attributed to a preference of As and Te to have Te atoms on their third and forth coordination shells. Hence, the picture of glassy $\mathrm{AsTe}_{3}$ could be rationalized in terms of highly chemically disordered structure (occurrence of As-As and Te-Te bonds) where As-centered motifs are linked through relatively long Te chains.

Let us now compare atomic bond lengths found in amorphous $\mathrm{AsTe}_{3}$ to those found in stable $\alpha-\mathrm{As}_{2} \mathrm{Te}_{3}$ and metastable $\beta-\mathrm{As}_{2} \mathrm{Te}_{3}$ crystalline counterparts. $\alpha-\mathrm{As}_{2} \mathrm{Te}_{3}$ and $\beta-\mathrm{As}_{2} \mathrm{Te}_{3}$ crystallize in monoclinic and rhombohedral phases, respectively. The crystal structure of $\alpha-\mathrm{As}_{2} \mathrm{Te}_{3}$ consists of zig-zag chains where As atoms are either trigonally (As(I) site) or octahedrally (As(II) site) coordinated to the Te atoms yielding three various $\mathrm{Te}$ ( $\mathrm{Te}(\mathrm{I}), \mathrm{Te}(\mathrm{II})$, and $\mathrm{Te}(\mathrm{III})$ ) sites. More precisely, Te(I) type atoms are bonded to two As(I) atoms (typical bond length $\mathrm{d}_{\mathrm{Te}(\mathrm{I})-\mathrm{As}(\mathrm{I})}=2.77 \AA$ ) and one $\mathrm{As}$ (II) atom $\left(\mathrm{d}_{\mathrm{Te}(\mathrm{I})-\mathrm{As}(\mathrm{II})}=2.93 \AA\right.$ ). $\mathrm{Te}$ (II) atoms are bonded to two As(II) atoms $\left(\mathrm{d}_{\mathrm{Te}(\mathrm{II})-\mathrm{As}(\mathrm{II})}=2.90 \AA\right.$ ) and one As(I) atom $\left(\mathrm{d}_{\mathrm{Te}(\mathrm{II})-\mathrm{As}(\mathrm{I})}=2.68 \AA\right)$ and finally, the Te(III) atoms are bonded to three As(II) atoms $\left(\mathrm{d}_{\mathrm{Te}(\mathrm{III})-\mathrm{As}(\mathrm{II})}=2.76\right.$ or $=2.85 \AA$ ) ${ }^{74}$ The $\beta-\mathrm{As}_{2} \mathrm{Te}_{3}$ compound is isostructural to the well-known $\mathrm{Bi}_{2} \mathrm{Te}_{3}$ based thermoelectric materials used in solid-state cooling applications. ${ }^{11 / 12}$ In this structure, As atoms exhibit a more homogeneous environment since all As atoms are octahedrally coordinated (three short As-Te bonds with $\mathrm{d}=2.735 \AA$ and three long As-Te bonds with $\mathrm{d}=3.132 \AA$ ). Overall, we remark that the AsTe bonds in both $\alpha$ - and $\beta$ - $\mathrm{As}_{2} \mathrm{Te}_{3}$ crystalline compounds are longer than those found in $\mathrm{As}_{2} \mathrm{Te}_{3}$ or $\mathrm{AsTe}_{3}$ glasses $\left(\mathrm{d}_{\mathrm{As}-\mathrm{Te}}=2.65\right.$ A).74.76

\subsection{Coordination numbers and atomic local environments}

More insights into the $\mathrm{AsTe}_{3}$ network can be obtained by looking at the atomic coordination numbers. Partial coordination numbers can be obtained by two different methods. The first method relies on the partial pair correlation functions and consists on estimating $n_{\alpha \beta}$ by integrating the first peak of $g_{\alpha \beta}(r)$ up to a given cutoff distance corresponding to the position of the first mini- 
mum. The total As and Te coordination numbers are then obtained as follows: $n_{\mathrm{As}}=n_{\mathrm{AsAs}}+n_{\mathrm{AsTe}}$, and $n_{\mathrm{Te}}=n_{\mathrm{TeTe}}+n_{\mathrm{TeAs}}$, respectively. We here note that $n_{\mathrm{AsTe}}=3 n_{\mathrm{TeAs}}$. Finally, the average coordination number can be defined irrespective of the chemical species and is given by $\bar{n}=c_{\mathrm{As}} n_{\mathrm{As}}+c_{\mathrm{Te}} n_{\mathrm{Te}}$. While this method is commonly used to estimate coordination numbers from molecular dynamics trajectories, its accuracy depends on the adopted distance cutoffs. In particular, in the case where first minimums of the $g_{\alpha \beta}(r)$ cannot be defined accurately, any small variation of the used cutoffs could lead to a significant change in the coordination numbers.

In order to go beyond this limitation, we resort to a second method based on the MLWF formalism. We recall that a Wannier function corresponds to the localization of two electrons and its center gives its average position. Hence, a chemical bond between two atoms $\alpha$ and $\beta$ is defined when a Wannier center $\mathrm{W}$ is shared between the two considered atoms such as $r_{\alpha \beta}-r_{\alpha W}-r_{\beta W} \leq 0.1 \AA$. Here $r$ is a distance, $\alpha$ and $\beta$ refers to As and/or Te atoms, and a $0.1 \AA$ tolerance is considered to account for small deviations that occurs on the spacial localization of the $\mathrm{W}$ center. Consequently, one can accurately estimate the coordination number of each element by counting the number of chemical bonds. In addition, proportion of atoms $\alpha$ (As or Te) that are $l$-fold coordinated to other atoms (As or/and Te), $\bar{n}_{\alpha}(l)$, can be obtained. An illustration of the Wannier centers occurring in $\mathrm{AsTe}_{3}$ is provided in Fig. 7

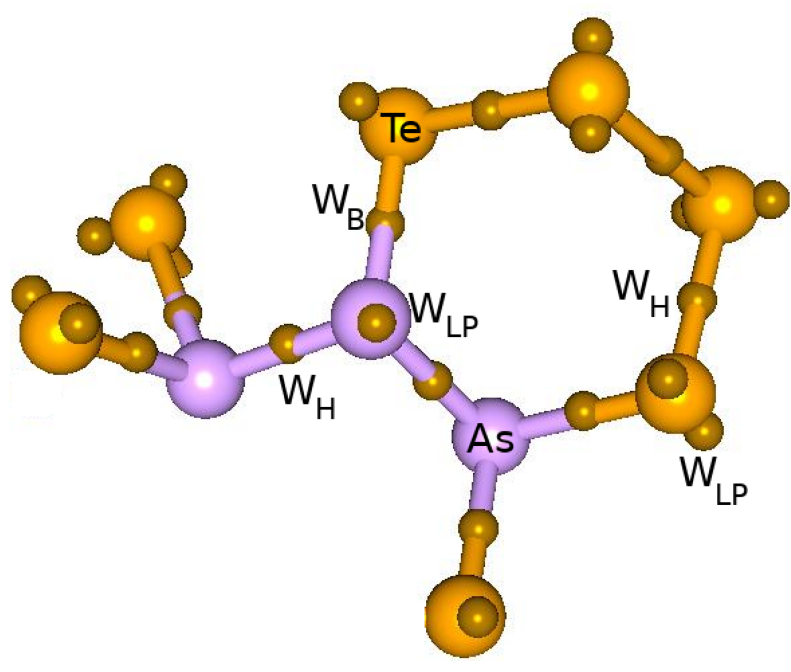

Fig. 7 Details of the bonding environment for glassy $\mathrm{AsTe}_{3}$. For the color code: As (purple), Te (light orange), and Wannier centers (labeled as W) (light brown). Only a few As and Te atoms are presented along with representative Wannier centers. We distinguish three different Wannier centers: $W_{B}$ centers occurring along As-Te bonds, $W_{H}$ centers occurring along homopolar $\mathrm{Te}-\mathrm{Te}$ or $\mathrm{As}-\mathrm{As}$ bonds, and $\mathrm{W}_{\mathrm{LP}}$, indicates the lone pair (LP) valence electrons not participating in chemical bonds.

Table 2 summarizes the obtained coordination numbers of $\mathrm{AsTe}_{3}$. We find that As and Te coordination numbers obtained by integration of $g_{\alpha \beta}(r)$ are slightly larger than those obtained from the MLFW analysis. In particular, $n_{\mathrm{TeTe}}$ shows the largest difference between the two methods, which can be ascribed to
Table 2 Coordination numbers as obtained from the FPMD and ML-GAP models. Results computed either by integrating the PDFs up to the first minimum or based on the MLWF analysis. Typical standard deviation of the MLWF results is of $\sim 0.02$.

\begin{tabular}{ccccc}
\hline \hline & \multicolumn{2}{c}{ FPMD } & \multicolumn{2}{c}{ ML-GAP } \\
\hline & PDF & MLWF & PDF & MLWF \\
$n_{\text {AsAs }}$ & 0.78 & 0.75 & 0.67 & 0.66 \\
$n_{\mathrm{AsTe}}$ & 2.26 & 2.25 & 2.36 & 2.32 \\
$n_{\mathrm{TeAs}}$ & 0.75 & 0.75 & 0.78 & 0.77 \\
$n_{\mathrm{TeTe}}$ & 1.33 & 1.25 & 1.45 & 1.31 \\
$n_{\mathrm{As}}$ & 3.04 & 3.00 & 2.98 & 2.99 \\
$n_{\mathrm{Te}}$ & 2.08 & 2.00 & 2.23 & 2.08 \\
\hline \hline
\end{tabular}

the ill defined first minimum in the $g_{\mathrm{TeTe}}(r)$. We now focus on the MLWF coordination numbers and compare the results of the FPMD and ML-GAP models. We find a slight dependence of the partial coordination numbers on the cell size. In particular, $n_{\mathrm{AsAs}}$ decreases from 0.75 (FPMD) to 0.66 (ML-GAP) at the expanses of $n_{\mathrm{AsTe}}$ that increases from 2.25 (FPMD) to 2.32 (ML-GAP). Nevertheless, the overall $n_{\mathrm{As}}$ remains constant (3.00) with increasing the cell size. Looking at $n_{\mathrm{TeAs}}$, we find that both models lead to a very similar values. In contrast, a larger $n_{\mathrm{TeTe}}$ is obtained from ML-GAP compared to FPMD. This leads to an $n_{\mathrm{Te}}$ of 2.08 in the case of the ML-GAP model and to 2.00 in the case of the FPMD model. Overall, the picture of the amorphous $\mathrm{AsTe}_{3}$ network as obtained from the MLWF formalism is in good agreement with the "8-N" rule which predicts coordination numbers of 3 and 2 for As and Te, respectively. These results are in good agreement with previous RMC data on $\mathrm{As}_{20} \mathrm{Te}_{80} \underline{17}$ and experiments. 1516

We deepen the description of the $\mathrm{AsTe}_{3}$ glassy network by looking at the details of the atomic local environments of As and $\mathrm{Te}$ atoms obtained through the MLWF decomposition on the FPMD and ML-GAP models (see Table 3). First we focus on the FPMD model. We find that $97 \%$ of As atoms are three-fold coordinated with a minor fraction of two- and four-fold As configurations (see table 3). Such a distribution of configurations is inline with the As coordination number $n_{\mathrm{As}}=3$. Looking at the breakdown of the 3 -fold As environment, we find that configurations containing at least one homopolar As-As bond (As-AsTe 2 and $\mathrm{As}-\mathrm{As}_{2} \mathrm{Te}$ ) are as numerous as $59 \%$ compared to the chemically ordered $\mathrm{As}^{-} \mathrm{Te}_{3}$ configurations (38\%). Interestingly, despite the low concentration of As atoms in $\mathrm{AsTe}_{3}, 61 \%$ of them belong to homopolar bonds (see Table 3). While this result agrees with previous modelling $17 \sqrt{18}$ it is in a strike contrast with NMR and Raman spectroscopy results ${ }^{15 \mid 16}$ where As-As homopolar bonds appear only for As concentration $x>0.3$ in amorphous $\mathrm{As}_{x} \mathrm{Te}_{1-x}$. This absence of As homopolar bonds in glassy $\mathrm{AsTe}_{3}$ might be ascribed to the way their fractions are estimated from NMR and Raman spectroscopy. Actually, both experiments ${ }^{15 / 16}$ rely on molecular dissociation models in order to estimate the fractions of As-Te, AsAs, and Te-Te bonds. On the one hand, this procedure employs empirical parameters and, on the other hand, it replies on the dissociation of either $\mathrm{As}_{2} \mathrm{Te}_{3}$ or AsTe. As such, the fraction of As-As bonds cannot be accessed in a straight forward manner and might be subject to large error bars due to the non inclusion of a more 
Table 3 Percentage $\bar{n}_{\alpha}(l)$ of the different coordination units in the FPMD model of glassy AsTe $e_{3}$, error bars are typically of $\sim 1 \%$. We note that the $n_{\alpha}(l)$ is given with respect to the number of atoms $\alpha$ in the system. We also provide the fractions of As and Te atoms involved in homopolar bonds $N_{\mathrm{AsAs}}$ and $N_{\mathrm{AsAs}}$, respectively. Values between parenthesis correspond to ML-GAP model.

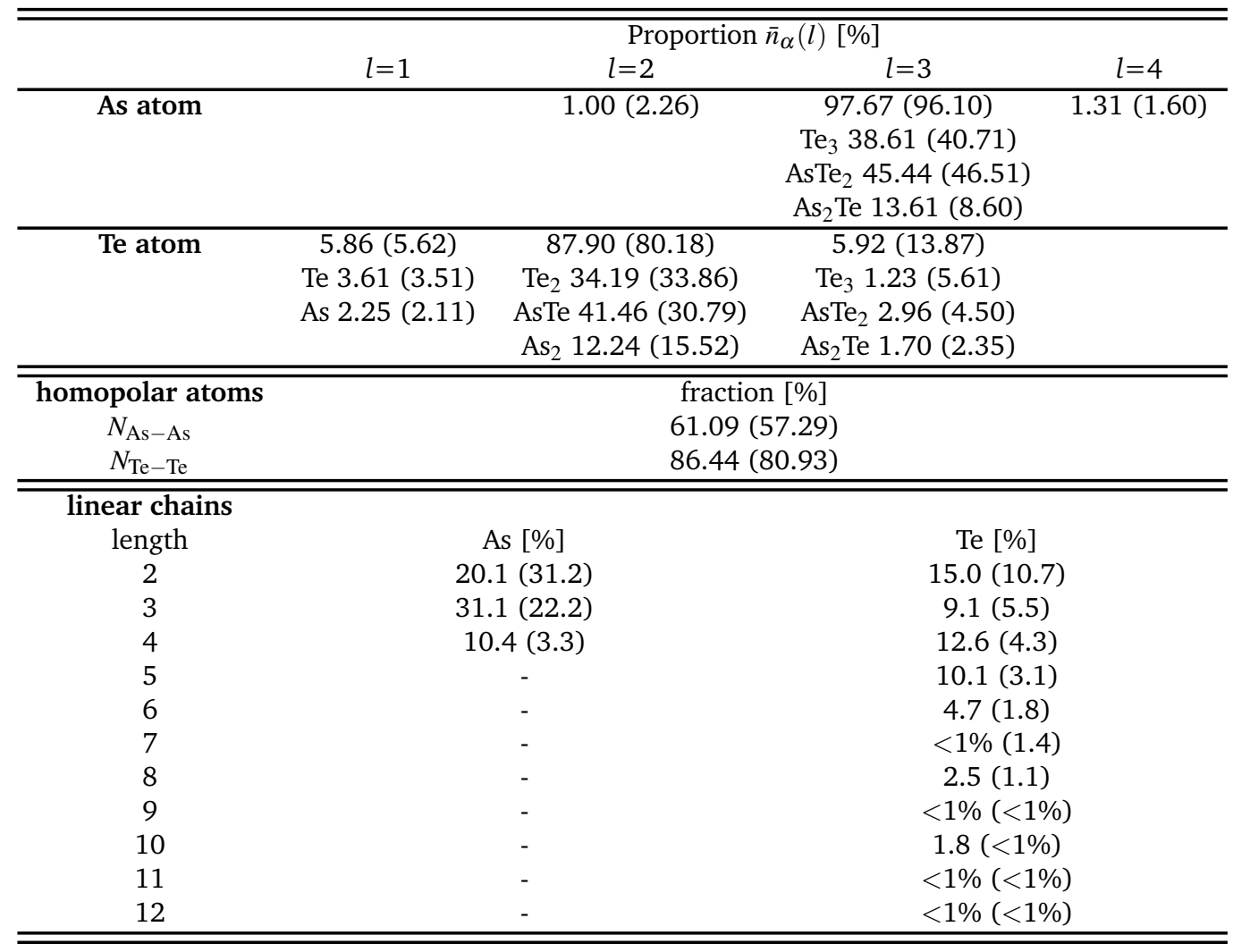


general model. Moreover, the occurence of large fractions of homopolar bonds in As-Te systems might also be ascribed to the very similar bond energies of As-As, As-Te, and Te-Te bonds. 77

Compared to As, Te shows a larger distribution of local environments. In particular, Te occurs mainly in 2-fold structural motifs $(87.9 \%)$ together with small fractions of under coordinated (1-fold Te, 5.8\%) and over coordinated (3-fold Te, 5.9 \%) units, in agreement with the Te coordination number $n_{\mathrm{Te}}=2$. Interestingly, while these three Te environments are present in crystalline $\mathrm{As}_{2} \mathrm{Te}_{3}, 16$ the occurrence of three fold coordinated $\mathrm{Te}$ in glassy $\mathrm{As}_{x} \mathrm{Te}_{1-x}$ has been longly discussed in literature. Recent EXAFS and NMR spectroscopy proved that Te atom is mainly two fold over the entire range of compositions as predicted by the 8-N rule. $\frac{16 \mid 17}{17}$ In the contrary, Mössbauer and Raman spectroscopy, ${ }^{15} \mid 28$ suggests the occurrence of a non negligible fraction of 3-fold Te. The picture of Te-rich amorphous $\mathrm{As}_{x} \mathrm{Te}_{1-x}$ as stems out from our FPMD model shows that a small fraction of 3-fold Te occurs in $\mathrm{AsTe}_{3}$. In particular, we find that 3-fold Te occurs in $\mathrm{Te}_{-} \mathrm{AsTe}_{2}$ (2.96 \%), $\mathrm{Te}_{-} \mathrm{As}_{2} \mathrm{Te}$ (1.7 \%), and, $\mathrm{Te}^{-\mathrm{Te}_{3}}$ (1.23 \%) units which differs from that found in the stoichiometric $\mathrm{As}_{2} \mathrm{Te}_{3}$ system where $\mathrm{Te}^{-\mathrm{As}_{3}}$ is the main building block.

Coming to the ML-GAP model, we remark that it reproduces the overall population of the atomic local environments found in the FPMD model. Nevertheless, few differences are noticeable and might be due to the enhanced description of the As-As correlations in the ML-GAP model. In particular, the fraction of As-As $s_{2} \mathrm{Te}$ in the ML-GAP model is found to decrease by about $5 \%$ compared to the FPMD model. This result is in line with the decrease of the $n_{\text {AsAs }}$ (see Table 2) discussed previously. More interestingly, we find that the fraction of 3-fold Te increases by about $8 \%$ at the expanses of 2-fold Te in the ML-GAP model with respect to the FPMD results. This finding consolidates our results on the presence of 3-fold $\mathrm{Te}$ in amorphous $\mathrm{AsTe}_{3}$ and shows that it occurs not only as a chemically ordered As- $\mathrm{Te}_{3}$ configuration, but also as As-AsTe ${ }_{2}$, and As-As 2 Te configurations. Such a picture might explain the difficulty of detecting Te(III) structural units from experiments. Furthermore, we find that the ML-GAP model features a sizeable fraction of homopolar bonds, $57 \%$ and $81 \%$ for As and $\mathrm{Te}$, respectively.

Overall, our results show that while As and Te fairly obey the 8-N rule, over and under coordinated atoms are present in glassy $\mathrm{AsTe}_{3}$. In the particular case of $\mathrm{Te}$, we find that the occurrence of $\mathrm{Te}(\mathrm{III})$ is not necessarily in contrast with an average coordination number close to $n_{\mathrm{Te}}=2$. Moreover, the 3 -fold Te environment is found to be altered by the presence of homopolar bonds.

\subsection{Network connectivity}

It is of interest to focus on the network topology and connectivity. Figure 8 shows the bond angle distributions around As and $\mathrm{Te}$ atoms. ML-GAP model shows smoother bond angle distributions than the FPMD system thanks to a larger number of As atoms. Therefore, we only comment the results of the ML-GAP model. As features a broad angular distribution centred around $\sim 95^{\circ}$ and $\sim 97^{\circ}$ in the case of As-As-Te and Te-As-Te environments, respectively, consistent with a pyramidal 3-fold structure of As. In
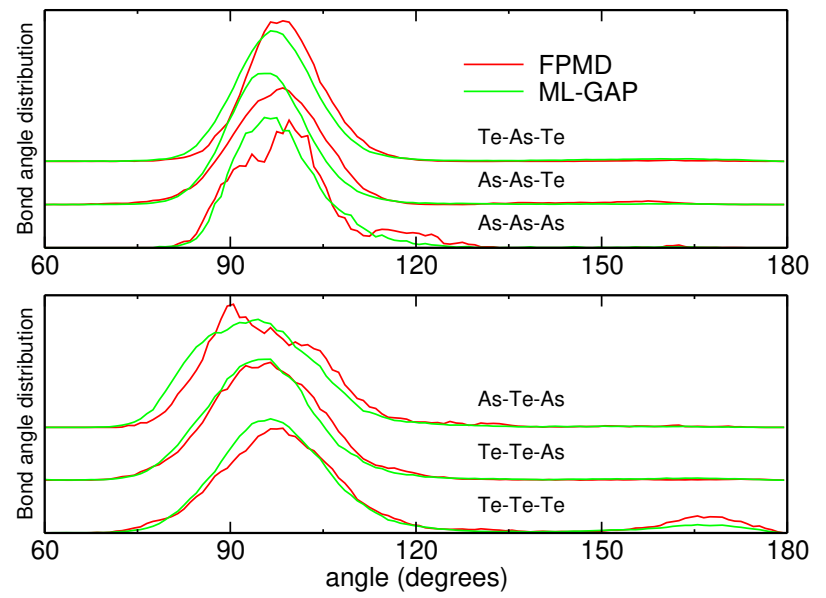

Fig. 8 Bond angle distribution around As atom (top) and around Te atom (bottom).

the case of As-As-As units we distinguish a main peak around $~$ $97^{\circ}$ and an extended tail up to $\sim 120^{\circ}$. Coming to Te, we find that As-Te-As bond angle distribution shows a broad peak centered at around $95^{\circ}$. In the case of Te-Te-As, the angular distribution becomes less broad and centers around $95^{\circ}$. Finally, for the TeTe-Te chains we distinguish a main peak centered around $97^{\circ}$ and a second less intense peak around $167^{\circ}$.

In order to access the structural origins of these peaks we report in Tab. 3 the chains analysis of glassy $\mathrm{AsTe}_{3}$. We note that a chain of atoms belonging to species $\alpha$ is defined as a linear sequence of atoms connected through chemical bonds where the two end atoms are singly coordinated, and the intermediate atoms are two fold coordinated to $\alpha$ atoms. In this definition, a chain of length $n$ is quantized by the number of atoms forming the chain normalized to the total number of atoms of species $\alpha$. We note that chains statistics obtained from the FPMD and the ML-GAP models cannot be directly compared as they contain substantially different fractions of $\mathrm{Te}_{-} \mathrm{Te}_{3}$ configurations, which lead to the exclusion of a sizeable number of Te atoms when searching for chains. Hereafter we solely focus on the results obtained from the large cell with ML-GAP.

Focusing on As, we remark that it forms mainly dimer and trimer chains, and to a less extent 4-membered As chains ( $\sim 3 \%)$. These chains are reflected in the As bond angle distribution. The first peak around $95-97^{\circ}$ is attributed to As pyramids irrespective of the chemical order and the tail observed in the As-As-As bond angle distribution in the vicinity of $\sim 118^{\circ}$ is attributed to As chains with $n=4$. The intensity of this peak is consistent with the small fraction of such chains. The As pyramids are predominantly linked to each other through three types of Te linkages. One can distinguish Te connecting two As pyramids is a "corner-sharing" fashion (As-Te-As), short Te chains involving Te dimers (As-Te-TeAs), and long Te chains (As-Te..Te..Te-As). As shown in Tab. 3 long linear Te chains up to $n=7$ occurs in relevant proportions and are responsible of the peak at $\sim 167^{\circ}$ in the Te-Te-Te bond angular distribution. 42

Aiming at a complete picture of glassy $\mathrm{AsTe}_{3}$ topology we in- 
vestigate the network connectivity through the rings analysis. We employ the RINGS method ${ }^{7879}$ to calculate the connectivity profile following the King/Franzblau shortest paths criterion. ${ }^{80 \mid 81}$ In this method, all atoms are considered as initial points to begin the search for a given ring, and homopolar bonds are allowed during the searching procedure. We use the first minimum of the partial pair correlation functions as cutoff distances and search for rings with a maximum of 30 atoms. The distribution of rings in $\mathrm{AsTe}_{3}$ can be quantified by two quantities: $\mathrm{R}_{c}(\mathrm{n})$ which represents the number of rings containing $\mathrm{n}$ atoms (As or Te), and $\mathrm{P}_{n}(\mathrm{n}$ ) represents the number of atoms that can be used as the origin of search for at least one ring containing $\mathrm{n}$ atoms. Both $\mathrm{R}_{c}(\mathrm{n})$ and $\mathrm{P}_{n}(\mathrm{n})$ are normalized to the total number of atoms in our model. Due to size effects, rings in the FPMD model show a large statistical fluctuations, therefore only results of the ML-GAP model are presented in Fig. 9

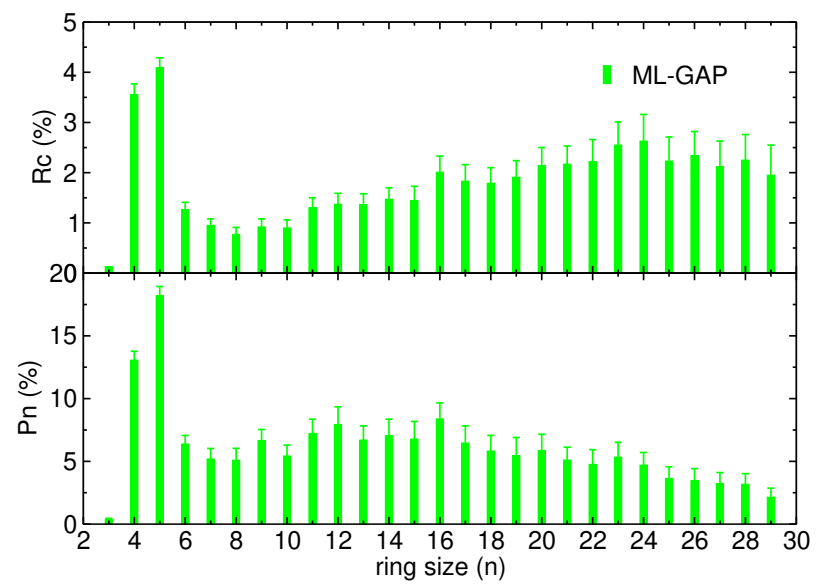

Fig. 9 Connectivity profiles for amorphous $\mathrm{AsTe}_{3}$ ML-GAP model obtained using the RINGS method. (top panel) $R_{c}(n)$, number of rings of size $\mathrm{n}$ normalized to the total number of atoms in the model, (bottom panel) $P_{n}(n)$, number of atoms at the origin of at least one ring of size $\mathrm{n}$ normalized to the total number of atoms in the model. Vertical lines represnts the standard deviation.

Our results reveal that only 4 and 5 membered rings show a meaningful concentration with about $13 \%$ and $18 \%$ atoms that could be at the origin of these rings, respectively.

In summary, the $\mathrm{AsTe}_{3}$ shows a particular connectivity where As belongs to pyramidal structures with a high level of chemical disorder. The pyramids are rather connected through Te chains than rings and no significant IRO can be identified in the amorphous phase of $\mathrm{AsTe}_{3}$. A representative snapshot of the $\mathrm{AsTe}_{3}$ structure is provided in Fig. 10

\section{Conclusions}

We used X-ray diffraction, first-principles molecular dynamics, and machine learning interatomic potentials to study the glassy phase of $\mathrm{AsTe}_{3}$ compound. X-ray diffraction experiments confirmed the nature of the amorphous phase $\mathrm{AsTe}_{3}$ elaborated by twin roller quenching technique. The differential scanning calorimetry showed that glassy $\mathrm{AsTe}_{3}$ exhibits a glass transition temperature $\mathrm{T}_{g}$ and a temperature of the onset of the crystal-

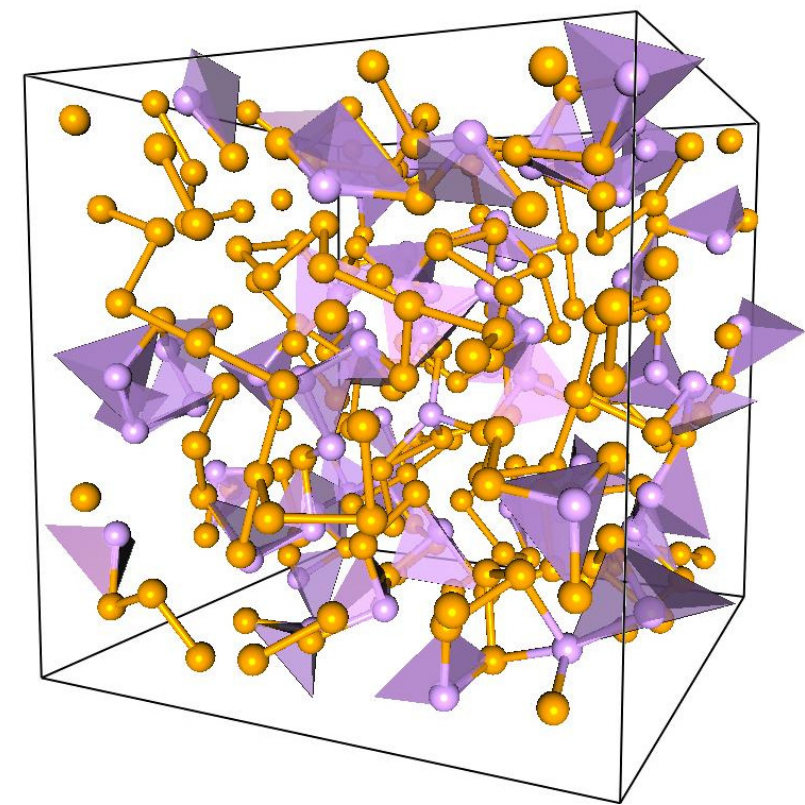

Fig. 10 Snapshot of amorphous $\mathrm{AsTe}_{3}$ FPMD model at $\mathrm{T}=300 \mathrm{~K}$. For the color code: As (purple), and Te (light green). As centered pyramids are also highlighted and periodic clones are shown for clarity.

lization $\mathrm{T}_{c}$ at $367 \mathrm{~K}$ and $413 \mathrm{~K}$, respectively. Measured X-ray total structure factor and total pair correlation function were compared to those obtained from the FPMD model yielding a very good agreement. We then exploit the FPMD date to generate a machine learned interatomic potential through the Gaussian approximation potential framework, and use it to produce a large size $\mathrm{AsTe}_{3}$ amorphous model. Then, the atomic scale structure of glassy $\mathrm{AsTe}_{3}$ was investigated based on the obtained FPMD and ML-GAP models. We found that As and Te obey the 8-N rule with an average coordination numbers of 3 and 2, respectively, in the case of the FPMD model, and 2.99 and 2.08, respectively, in the case of the ML-GAP model. In addition, we find that significant fractions of under and over coordinated atoms exist in the amorphous phase with about 6\% (FPMD) and 13\% (ML-GAP) of 3-fold Te. Despite the low As concentration in $\mathrm{AsTe}_{3}$, its local environment features a very high chemical disorder that manifests through the occurrence of homopolar bonds including $57 \%$ of As atoms (ML-GAP model). The overall picture of $\mathrm{AsTe}_{3}$ glass is mainly made by As centered pyramidal structures linked through $\mathrm{Te}_{n}$ chains. Our results provides a comprehensive picture of the chemistry and network connectivity of amorphous $\mathrm{AsTe}_{3}$.

\section{Acknowledgments}

The authors thank Mickaël Bigot for his help in sample preparation and Sébastien Le Roux for his courtesy on providing Atomes software (https://www.conectus.fr/atomes) that was used for part of the analysis and for making Figs. 7 and 10. We used computational resources provided by the computing facilities MCIA (Mésocentre de Calcul Intensif Aquitain) of the Université de Bordeaux and of the Université de Pau et des Pays l'Adour. 


\section{Notes and references}

1 S. Kondo, K. Takada and Y. Yamamura, Solid State Ion., 1992, 53, 1183-1186.

2 Y. Seino, T. Ota, K. Takada, A. Hayashi and M. Tatsumisago, Energy Environ. Sci., 2014, 7, 627-631.

3 T. Hakari, M. Nagao, A. Hayashi and M. Tatsumisago, J. Power Sources, 2015, 293, 721-725.

4 J. Sanghera and I. Aggarwal, J. Non-Cryst. Solids, 1999, 256, 6-16.

5 M. Wuttig, Phys. Status Solidi B, 2012, 249, 1843-1850.

6 W. Zhang, V. L. Deringer, R. Dronskowski, R. Mazzarello, E. Ma and M. Wuttig, MRS Bulletin, 2015, 40, 856-869.

7 P. Dos Santos, M. De Araujo, A. Gouveia-Neto, J. Medeiros Neto and A. Sombra, Appl. Phys. Lett., 1998, 73, $578-580$

8 B. Bureau, X. H. Zhang, F. Smektala, J.-L. Adam, J. Troles, H.1. Ma, C. Boussard-Plèdel, J. Lucas, P. Lucas, D. Le Coq et al., Journal of non-crystalline solids, 2004, 345, 276-283.

9 J. D. Musgraves, J. Hu and L. Calvez, Springer Handbook of Glass, Springer, 2019.

10 X. Zhang, Y. Guimond and Y. Bellec, J. Non-Cryst. Solids, 2003, 326, 519-523.

11 H. Goldsmid, Thermoelectric refrigeration, Springer, 2013.

12 D. M. Rowe, Thermoelectrics and its energy harvesting. 2. Modules, systems, and applications in thermoelectrics, CRC, 2012.

13 J.-B. Vaney, J. Carreaud, G. Delaizir, A. Pradel, A. Piarristeguy, C. Morin, E. Alleno, J. Monnier, A. P. Gonçalves, C. Candolfi et al., Adv. Electron. Mater., 2015, 1, 1400008.

14 J.-B. Vaney, J. Carreaud, A. Piarristeguy, C. Morin, G. Delaizir, R. Viennois, M. Colas, J. Cornette, E. Alleno, J. Monnier et al., Inorg. Chem., 2018, 57, 754-767.

15 A. Tverjanovich, K. Rodionov and E. Bychkov, J. Solid State Chem., 2012, 190, 271-276.

16 D. C. Kaseman, I. Hung, K. Lee, K. Kovnir, Z. Gan, B. Aitken and S. Sen, J. Phys. Chem. B, 2015, 119, 2081-2088.

17 P. Jóvári, S. Yannopoulos, I. Kaban, A. Kalampounias, I. Lishchynskyy, B. Beuneu, O. Kostadinova, E. Welter and A. Schöps, J. Chem. Phys., 2008, 129, 214502.

18 Q. Ma, D. Raoux and S. Benazeth, Phys. Rev. B, 1993, 48, 16332.

19 J. Cornet and D. Rossier, J. Non-Cryst. Solids, 1973, 12, 6184.

20 N. S. Platakis, J. Non-Cryst. Solids, 1977, 24, 365-376.

21 P. Eusner, L. Durden and L. Slack, J. Am. Ceram. Soc., 1972, $55,43-46$.

22 R. K. Quinn, Mater. Res. Bull., 1974, 9, 803-813.

23 J. Savage, J. Non-Cryst. Solids, 1972, 11, 121-130.

24 S. Sen, S. Joshi, B. Aitken and S. Khalid, J. Non-Cryst. Solids, 2008, 354, 4620-4625.

25 S. Titus, S. Asokan and E. Gopal, Solid State Commun., 1992, 83, 745-747.

26 S. Titus, S. Asokan, T. Panchapagesan and E. Gopal, Phys. Rev. B, 1992, 46, 14493.
27 S. Titus, R. Chatterjee, S. Asokan and A. Kumar, Phys. Rev. B, 1993, 48, 14650.

28 M. Tenhover, P. Boolchand and W. Bresser, Phys. Rev. B, 1983, 27, 7533 .

29 A. Bouzid, S. Le Roux, G. Ori, M. Boero and C. Massobrio, J. Chem. Phys., 2015, 143, 034504.

30 S. Le Roux, A. Bouzid, M. Boero and C. Massobrio, J. Chem. Phys., 2013, 138, 174505.

31 M. Celino, S. Le Roux, G. Ori, B. Coasne, A. Bouzid, M. Boero and C. Massobrio, Phys. Rev. B, 2013, 88, 174201.

32 K. Wezka, A. Bouzid, K. J. Pizzey, P. S. Salmon, A. Zeidler, S. Klotz, H. E. Fischer, C. L. Bull, M. G. Tucker, M. Boero, S. Le Roux, C. Tugène and C. Massobrio, Phys. Rev. B, 2014, 90, 054206.

33 S. Le Roux, A. Bouzid, M. Boero and C. Massobrio, Phys. Rev. $B, 2012,86,224201$.

34 A. Bouzid, H. Zaoui, P. L. Palla, G. Ori, M. Boero, C. Massobrio, F. Cleri and E. Lampin, Phys. Chem. Chem. Phys., 2017, 19, 9729-9732.

35 G. Ori, C. Massobrio, A. Bouzid, M. Boero and B. Coasne, Phys. Rev. B, 2014, 90, 045423.

36 A. Pradel, T. Pagnier and M. Ribes, Solid State Ion., 1985, 17, 147-154.

37 A. Hrubỳ, Czechoslovak Journal of Physics B, 1972, 22, 11871193.

38 O. Masson, pyTSRedX, a data reduction program to obtain the atomic pair distribution function (PDF) from X-ray total scattering data, 2018.

39 A. D. Becke, Phys. Rev. A, 1988, 38, 3098.

40 C. Lee, W. Yang and R. G. Parr, Phys. Rev. B, 1988, 37, 785.

41 J. P. Perdew, K. Burke and M. Ernzerhof, Phys. Rev. Lett, 1996, 77, 3865-3868.

42 A. Bouzid, C. Massobrio, M. Boero, G. Ori, K. Sykina and E. Furet, Phys. Rev. B, 2015, 92, 134208.

43 A. Bouzid, G. Ori, M. Boero, E. Lampin and C. Massobrio, Phys. Rev. B, 2017, 96, 224204.

44 A. Bouzid, S. Gabardi, C. Massobrio, M. Boero and M. Bernasconi, Phys. Rev. B, 2015, 91, 184201.

45 Z. Chaker, G. Ori, M. Boero, C. Massobrio, E. Furet and A. Bouzid, J. Non-Cryst. Solids, 2018, 498, 338-344.

46 N. Troullier and J. L. Martins, Phys. Rev. B, 1991, 43, 8861.

47 S. Grimme, J. Comput. Chem., 2006, 27, 1787-1799.

48 J. Hutter, M. Parrinello, D. Marx, P. Focher, M. Tuckerman, W. Andreoni, A. Curioni, E. Fois, U. Röthlisberger, P. Giannozzi et al., IBM Zurich Research Laboratory and MPI für Festkörperforschung, 1995, 2001,.

49 S. Nosé, Mol. Phys., 1984, 52, 255-268.

50 S. Nosé, J. Chem. Phys., 1984, 81, 511-519.

51 W. G. Hoover, Phys. Rev. A., 1985, 31, 1695-1697.

52 G. J. Martyna, M. L. Klein and M. Tuckerman, J. Chem. Phys., 1992, 97, 2635-2643.

53 P. E. Blöchl and M. Parrinello, Phys. Rev. B, 1992, 45, 9413.

54 A. Bouzid, K. J. Pizzey, A. Zeidler, G. Ori, M. Boero, C. Massobrio, S. Klotz, H. E. Fischer, C. L. Bull and P. S. Salmon, Phys. 
Rev. B, 2016, 93, 014202.

55 A. Bouzid and C. Massobrio, J. Chem. Phys., 2012, 137, 046101.

56 R. Resta and S. Sorella, Phys. Rev. Lett., 1999, 82, 370-373.

57 N. Marzari and D. Vanderbilt, Phys. Rev. B, 1997, 56, 1284712865.

58 A. P. Bartók, M. C. Payne, R. Kondor and G. Csányi, Phys. Rev. Lett., 2010, 104, 136403.

59 W. J. Szlachta, A. P. Bartók and G. Csányi, Phys. Rev. B, 2014, 90, 104108.

60 A. P. Bartók and G. Csányi, International Journal of Quantum Chemistry, 2015, 115, 1051-1057.

61 D. J. MacKay and D. J. Mac Kay, Information theory, inference and learning algorithms, Cambridge university press, 2003.

62 C. E. Rasmussen and H. Nickisch, The Journal of Machine Learning Research, 2010, 11, 3011-3015.

63 F. C. Mocanu, K. Konstantinou, T. H. Lee, N. Bernstein, V. L. Deringer, G. Csanyi and S. R. Elliott, J. Chem. Phys. B, 2018, 122, 8998-9006.

64 P. Rowe, V. L. Deringer, P. Gasparotto, G. Csányi and A. Michaelides, J. Chem. Phys., 2020, 153, 034702.

65 D. Dragoni, T. D. Daff, G. Csányi and N. Marzari, Phys. Rev. Mater., 2018, 2, 013808.

66 G. Sivaraman, A. N. Krishnamoorthy, M. Baur, C. Holm, M. Stan, G. Csányi, C. Benmore and Á. Vázquez-Mayagoitia, npj Computational Materials, 2020, 6, 1-8.

67 A. P. Bartók, R. Kondor and G. Csányi, Physical Review B, 2013, 87, 184115.

68 S. Plimpton, J. Comp.Phys., 1995, 117, 1-19.

69 T. E. Faber and J. M. Ziman, Philosophical Magazine, 1965, 11, 153-173.

70 O. Masson and P. Thomas, J. Appl. Crystallogr, 2013, 46, 461465.

71 P. S. Salmon, J. Non-Cryst. Solids, 2007, 353, 2959-2974.

72 S. R. Elliott, Nature, 1991, 354, 445.

73 V. Brazhkin, E. Bychkov and O. Tsiok, Phys. Rev. B, 2017, 95, 054205.

74 G. J. Carron, Acta Crystallogr., 1963, 16, 338-343.

75 H. W. Shu, S. Jaulmes and J. Flahaut, Materials research bulletin, 1986, 21, 1509-1514.

76 V. P. Cuenca-Gotor, J. Sans, J. Ibáñez, C. Popescu, O. Gomis, R. Vilaplana, F. Manjón, A. Leonardo, E. Sagasta, A. SuárezAlcubilla et al., J. Phys. Chem. C, 2016, 120, 19340-19352.

77 M. Deli, D. H. Boigny and G. Kra, J. Non-Cryst. Solids, 2010, 10, 59-65.

78 S. Le Roux and P. Jund, Comput. Mater. Sci., 2010, 49, 70-83.

79 S. L. Roux and P. Jund, Comput. Mater. Sci., 2011, 50, 12171217.

80 S. V. King, Nature, 1967, 213, 1112-1113.

81 D. Franzblau, Phys. Rev. B, 1991, 44, 4925. 